$6 \quad$ Hongwei Zhou, $\mathrm{PhD}^{1,2, \#}$
8

\title{
Airway bacterial and fungal microbiome in chronic obstructive
}

\section{pulmonary disease}

Haiyue Liu, $\mathrm{PhD}^{1,2, *}$; Zhenyu Liang, $\mathrm{MD}^{3, *}$; Nannan Cao, $\mathrm{MD}^{1,2, *}$; Xilan Tan, $\mathrm{PhD}^{1,2}$;

Zuheng Liu, MD ${ }^{4}$; Fengyan Wang, $\mathrm{MD}^{3}$; Yuqiong Yang, $\mathrm{MSc}^{3}$; Chunxi Li, MSc ${ }^{5}$;

Yan $\mathrm{He}, \mathrm{PhD}^{1,2}$; Jin Su, $\mathrm{MD}^{5}$; Rongchang Chen, $\mathrm{MD}^{3}$; Zhang Wang, $\mathrm{PhD}^{2,6, \# \text {; }}$

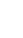

${ }^{1}$ State Key Laboratory of Organ Failure Research, Division of Laboratory Medicine, Zhujiang Hospital, Southern Medical University, Guangzhou, China.

${ }^{2}$ Microbiome Medicine Center, Zhujiang Hospital, Southern Medical University, Guangzhou, China.

${ }^{3}$ State Key Laboratory of Respiratory Disease, National Clinical Research Center for Respiratory Disease, Guangzhou Institute of Respiratory Health, the First Affiliated Hospital of Guangzhou Medical University, Guangzhou, China.

${ }^{4}$ State Key Laboratory of Organ Failure Research, Department of Cardiology, Nanfang Hospital, Southern Medical University, Guangzhou, China.

${ }^{5}$ Chronic Airways Diseases Laboratory, Department of Respiratory and Critical Care Medicine, Nanfang Hospital, Southern Medical University, Guangzhou, China.

${ }^{6}$ Institute of Ecological Science, School of Life Science, South China Normal University, Guangzhou, China.

\footnotetext{
* These authors contributed equally.
} 
$23 \quad$ \#Address correspondence to Hongwei Zhou, biodegradation@gmail.com and Zhang

24 Wang,wangz@m.scnu.edu.cn.

25

26 Running title: COPD airway bacterial-fungal microbiota

27 
Abbreviation List: AUC: Area under the curve; COPD: Chronic obstructive pulmonary disease; ENA: European Nucleotide Archive; FDR: False discovery rate; FEV1: Forced expiratory volume in $1 \mathrm{~s}$; FVC: Forced vital capacity; GOLD: Global Initiative for Chronic Obstructive Lung Disease; LDA: Linear discriminant analysis; LEfSe: Linear discriminant analysis effect size; IL: Interleukin; INF- $\gamma$ : Interferon- $\gamma$; OTUs: Operational taxonomic units; PBS: phosphate-buffered saline; PCoA: Principal Coordinate Analysis; QIIME: Quantitative Insights into Microbial Ecology; sRAGE: Soluble receptor for advanced glycation endproducts; TGF- $\beta$ : Transforming growth factor- $\beta$; TNF- $\alpha$ : Tumor necrosis factor $\alpha$; VEGF: Vascular endothelial growth factor. 


\section{Abstract}

Background: Little is known about airway mycobiome, and its relationship with bacterial microbiome in chronic obstructive pulmonary disease (COPD).

Methods: Here we report the first simultaneous characterization of sputum bacterial and fungal microbiome in 84 stable COPD and 29 healthy subjects, using $16 \mathrm{~S}$ ribosomal DNA and fungal internal transcribed spacer DNA sequencing.

Results: Ascomycota predominated over Basidiomycota in fungal microbiome both in COPD patients and healthy controls. Meyerozyma, Candida, Aspergillus and Schizophyllum were most abundant at the genus level. There was a significant inverse correlation between bacterial and fungal microbial diversity, both of which altered in opposite directions in COPD patients versus controls, and in frequent versus non-frequent exacerbators. An enhanced bacterial-fungal ecological interaction was observed in COPD patients, which was characterized by higher proportion of co-occurrence intrakingdom interactions and co-exclusive interkingdom interactions. In COPD, four mutually co-occurring fungal operational taxonomic units (OTUs) in Candida palmioleophila, Aspergillus and Sordariomycetes exhibited co-exclusive relationships with other fungal OTUs, which was specifically present in frequent exacerbators but not in non-frequent exacerbators. Conversely, the mutual co-occurrence interactions between bacterial OTUs in Rothia mucilaginosa, Streptococcus, Veillonella and Prevotella, showed up in non-frequent exacerbators 
62

64

but not in frequent exacerbators. The perturbed bacterial-fungal interactions in COPD were associated with increased airway inflammatory mediators such as IL-6 and IL-8.

Interpretation: The disruption of airway bacterial-fungal community balance, characterized by the loss of commensal bacterial taxa and enriched pathogenic fungal taxa, is implicated in COPD. The airway mycobiome is an important cofactor mediating COPD pathogenic infection and host inflammation.

Clinical Trial Registration: www.clinicaltrials.gov (NCT 03240315).

Keywords: COPD, frequent exacerbator, airway microbiome, mycobiome, bacterial-fungal interactions 


\section{Introduction}

Chronic obstructive pulmonary disease (COPD) is characterized by chronic airway inflammation resulting in irreversible decline in respiratory function and capacity. Bacterial, fungal and viral infections drive airway inflammation, and are associated with poorer disease outcome(1) and declined lung function(2, 3). The airway microbiome, the collective airway microbial community, is hypothesized to mediate the interactions between pathogenic infection and host inflammatory response $(4,5)$. Through interacting with bacteria and mucosal immune system(6), the fungal community can be a cofactor for airway inflammation and COPD progression(7). Essentially all previous airway microbiome studies, however, have focused on bacterial community in $\operatorname{COPD}(4,8-14)$. The non-bacterial members of airway microbiome in particular the fungal microbiome (or mycobiome), despite being of clinical relevance, have been largely underappreciated(7). Recent studies have reported the airway fungal composition in asthma(15), bronchiectasis(16), cystic fibrosis(17, 18), HIV(19) and lung transplantation(20); however, little is known on the mycobiome in COPD. The ecological interaction between airway bacterial and fungal microbiome and its role in COPD pathogenesis remains unexplored.

Susceptibility to frequent exacerbations represents an independent clinical phenotype in COPD, the 'frequent exacerbator' phenotype, and is associated with poorer clinical outcome $(21,22)$. The Global Initiative for Chronic Obstructive Lung Disease (GOLD) 2019 has redefined the measure of disease severity to recognized the high 
exacerbation risk(23) (>=2 exacerbations and /or 1 hospitalization in the previous year). The pathophysiology underlying the frequent exacerbation phenotype is manifested by an interplay between enhanced airway immune responses, bacterial and fungal colonization and dynamic lung hyperinflation, that together predispose patients to persistent inflammation and recurrent exacerbations(22). Identifying markers that predict patient exacerbation frequency is of great importance for COPD management. Difference in baseline respiratory microbiome composition was hypothesized to explain the different exacerbation frequency in COPD patients(21). However, studies assessing baseline airway microbiome have not found significant differences in bacterial composition between frequent and non-frequent exacerbators $(4,14)$. Recent longitudinal studies suggested that temporal variability of the sputum microbiome could be associated with COPD exacerbation frequency $(14,24)$. However, measuring microbial temporal variability requires serial sampling of sputum microbiome in multiple timepoints and is therefore not clinically practical. Assessing the airway fungal microbiome might open up opportunities in identifying novel markers for the frequent exacerbator phenotype.

Here we characterized the airway bacterial and fungal microbiome simultaneously in clinically stable COPD patients. We hypothesize that the ecological interactions between bacterial, fungal microbiome and host inflammation are associated with disease and exacerbation frequency. We showed that bacterial and fungal microbiome co-altered in COPD. The perturbation of bacterial-fungal interactions in COPD was 
118

119

associated with host inflammation and the frequent exacerbator phenotype.

\section{Materials and Methods}

\section{Subjects and samples}

Sputum samples of 113 individuals, including 84 stable COPD patients and 29 healthy controls, were collected in the First Affiliated Hospital of Guangzhou Medical University. COPD patients were divided into frequent (FE: exacerbation events $>=2$ or 1 hospitalization due to exacerbation of $\mathrm{COPD} /$ past year) and non-frequent exacerbators (NE). The study was approved by the ethics committee of the First Affiliated Hospital of Guangzhou Medical University and was registered in www.clinicaltrials.gov (NCT 03240315). All subjects provided written informed consent in accordance with the Declaration of Helsinki.

\section{Bacterial and fungal microbiome sequencing}

Bacterial genomic DNA was extracted from selected sputum plugs using a Total Nucleic Acid Extraction Kit (Bioeasy Technology, Inc., Shenzhen, China) as per the manufacturer's instructions. Negative controls for extraction (no sputum) and PCR amplification (no DNA template, $\mathrm{ddH}_{2} \mathrm{O}$ only) were included in each experiment. The extraction negative controls were subsequently sequenced to identify any potential contaminating bacterial/fungal species. The V4 hypervariable region of bacterial $16 \mathrm{~S}$ rRNA gene and fungal 18S-28S rRNA gene internally transcribed spacer region ITS1 DNA were amplified using barcoded primers, and were sequenced using iTorrent sequencing platform. 


\section{Sequence processing and analysis}

Sequence processing and analysis were performed using QIIME 1.9.1(25). The

143 obtained sequences were de-multiplexed, trimmed of barcodes and primers, and

filtered if they contained ambiguous bases or mismatches in the primer regions, according to the BIPES protocol(26). Chimeras were filtered out using UCHIME in de novo mode(27). After quality filtering and chimera removal, $16 \mathrm{~S}$ rRNA gene

147 sequencing resulted in a median read depth of 4,380, ITS1 DNA sequencing resulted 148 in a median read depth of 9,783. Both $16 \mathrm{~S}$ rRNA V4 region and ITS1 DNA 149 sequencing data of all subjects were subsampled to a uniform depth of 1,000 reads based on rarefaction curve asymptotes and Good's coverage values. Comparable rarefaction depth has been used in airway microbiome analyses $(4,28)$.

High-quality sequence reads for bacterial 16S rRNA V4 and fungal ITS1 region were

154 clustered into operational taxonomic units (OTUs) using USEARCH v11(29) in de novo mode with $97 \%$ sequence similarity cutoff. The taxonomy of representative $16 \mathrm{~S}$ rRNA gene sequences were determined using PyNAST with the Greengenes 13_8 157 database as reference(30). The taxonomy of representative ITS sequences were 158 determined using the QIIME_ITS database as reference (version information: 159 sh_qiime_release_s_28.06.2017)(25). The taxonomy of highly abundant unclassified 160 fungal OTUs was further determined using the phylotyping algorithm in MEGAN5 

downstream analysis. The sequences were deposited in the European Nucleotide Archive (ENA) under accession numbers PRJEB27507.

The biome data were filtered using the filter_otus_from_otu_table.py script with the parameter (-s 3) to remove low-abundance OTUs. Fifty-four OTUs (28 bacterial and 16 fungal OTUs) were selected that were $>0.5 \%$ average abundance for the major of OTU-based analysis. Airway microbial alpha diversity (diversity within samples) was

172 calculated using the Shannon indices. Airway microbial beta diversity (composition

173 dissimilarity between samples) was determined by using the unweighted UniFrac

174 distance and visualized in Principal Coordinate Analysis (PCoA). Adonis was used to

175 estimate statistical significance. Differential features between groups were identified 176 using a linear discriminant analysis (LDA) effect size (LEfSe) method with a

177 threshold of logarithmic LDA score 2.0(32). Random forest analysis was performed

178 using the OTU data selected by LEfSe using Weka 3.8

179 (https://www.cs.waikato.ac.nz/ml/weka/) with a 7-fold cross-validation(33).

Co-occurrence and co-exclusion relationships between the 54 abundant bacterial and

181 fungal OTUs were estimated using SparCC algorithm(34), known for its robustness to

182 compositional effects in microbiome dataset. The p-value was estimated by 100

183 bootstraps and the correlations with $\mathrm{p}<0.05$ were retained. Association between 
184

185

bacterial and fungal OTUs and inflammatory mediators was assessed using Spearman $\begin{array}{lllll}\text { correlation. Network was visualized using } & \text { Cytoscape }\end{array}$ (https://cytoscape.org/)(35). The false discovery rate (FDR)(36) method was used to adjust $P$-values for multiple testing wherever applicable.

\section{Results}

$\underline{\text { Overview of sputum bacterial and fungal microbiome }}$

Sputum samples were collected from 84 stable COPD patients and 27 healthy controls (Table 1, Table S1). Consistent with previous studies $(4,8-11,14,24,37)$, the majority of bacterial taxa belongs to Firmicutes (28.3\%), Bacteroidetes (27.2\%), Proteobacteria (24.6\%), Actinobacteria (7.0\%) and Fusobacteria (5.7\%) at the phylum level. For the fungal microbiome, $76.6 \%$ sequences belong to Ascomycota (71.7\%) or Basidiomycota $(4.9 \%)$ and $17.7 \%$ sequences to unidentified fungi taxa. The most abundant fungal genera are Meyerozyma, Candida, Aspergillus and Schizophyllum (>1\% average) (Fig. 1). There was a significant negative correlation between bacterial and fungal alpha diversity (Shannon, Spearman's rho=-0.172, $\mathrm{p}=0.04$ ). No significant association was found between bacterial or fungal microbiome with age, gender, smoking status and predicted FEV1\% (Fig. 1).

\section{Sputum bacterial and fungal microbiome in COPD patients and healthy controls}

The bacterial composition shifted in COPD patients compared to healthy subjects, with a slight decrease in alpha diversity (Fig. 2a) and a significant decreased 
abundance of genera Streptococcus, Peptostrptococcus, Porphyromonas, Lautropia and Actinomyces (Wilcoxon, FDR $\mathrm{p}<0.05$, Fig. 1). Conversely, the fungal diversity significantly increased in COPD (Fig. 2b), with a significant increased abundance in Candida and Schizophyllum, and three unclassified genera in Sordariomycetes, Saccharomycetales and Hypocreales (Wilcoxon, FDR p<0.05, Fig. 1). Beta-diversity analysis indicated a better separation between healthy and COPD groups using fungal than bacterial composition (Adonis, bacteria: $\mathrm{R}^{2}=0.016, \mathrm{p}<0.01$; fungi: $\mathrm{R}^{2}=0.061$, p<0.01, Fig. 2a-b). LEfSe analysis identified seven bacterial OTUs (bOTUs) and seven fungal OTUs (fOTUs) associated with disease state (LDA>2.0, Fig. S1-2). Random forest analysis discriminated COPD patients from controls with an area under the curve (AUC) of $0.83,0.91$ and 0.97 using these bacterial, fungal and their combined OTUs, respectively (Fig. 2c). Sub-analysis using 52 age, gender and smoking-status matched subjects indicated that the observed microbiome differences were not related to these factors (Table S2, Fig. S3).

\section{$\underline{\text { Sputum bacterial and fungal microbiome in frequent and non-frequent exacerbators }}$}

COPD patients were divided into frequent (FE: exacerbation events $\geq 2$ or 1 hospitalization due to exacerbation of $\mathrm{COPD}$ /past year) and non-frequent exacerbators (NE). Patient demographic factors are overall comparable between the two groups except for a significantly higher FEV1\% predicted in NE group (Table 1). Bacterial alpha diversity was significantly higher (Fig. 3a) in FE compared to NE group, whereas fungal alpha diversity showed the opposite trend (Fig. 3b). Veillonella was 
significantly decreased in FE group (Wilcoxon, FDR p<0.05, Fig. 1), whereas fungal genera Candida was significantly increased. Beta-diversity analysis also indicated a better separation between FE and NE for fungal compared to bacterial composition (Adonis, bacteria: $\mathrm{R}^{2}=0.019, \mathrm{p}<0.01$; fungi: $\mathrm{R}^{2}=0.046$, $\mathrm{p}<0.01$, Fig. 3a-b). Seven bOTUs and seven fOTUs were associated with exacerbation frequency using LEfSe (LDA>2.0, Fig. S4-5). Random forest analysis showed an AUC value of 0.78, 0.74 and 0.81 in separating the two groups using these bacterial, fungal and their combined OTUs respectively (Fig. 3c).

\section{Bacterial-fungal interactions in COPD patients and healthy controls}

To explore ecological interactions between airway bacterial and fungal microbiome, we performed network analyses using 54 bacterial and fungal OTUs $(>0.5 \%$ average relative abundance) using the SparCC algorithm(34). We observed considerable differences in bacterial-fungal interactions between COPD patients and controls. For COPD patients, 244 significant correlation pairs comprising of 144 bacteria-bacteria (B-B), 32 fungi-fungi (F-F) and 68 bacteria-fungi (B-F) interactions were identified (Fig. 4a-b, Fig. S6, p<0.05). Among them, 100 (69.4\%) B-B, 23 (71.9\%) F-F and 21 $(30.9 \%)$ B-F correlations were positive, indicating predominant co-occurring intrakingdom interactions and co-exclusive interkingdom interactions in COPD (Fig. 4a). Among the inverse relationships between bacterial and fungal OTUs, the correlations between bOTU2 Prevotella melaninogenica and fOTU15 Leucosporidium scottii, and between bOTU22 Veillonella dispar and fOTU2 Candida 
250

251

palmioleophila were most significant (Table S3-4). In comparison, the same analysis yielded a reduced interaction network for healthy controls with 86 significant correlations, the majority of which $(78,90.7 \%)$ were B-B interactions (Fig. 4a-b). To adjust for sample size, we reconstructed disease network using a balanced sample size with healthy subjects $(n=29)$. Despite a relatively smaller network, the network topology generally resembled that using all subjects (Fig. 4a, Fig. S7a), indicating sample size was likely not confounding the different networks between the two groups.

\section{Bacterial-fungal interactions in frequent and non-frequent exacerbators}

We further performed sub-analysis on the interaction network for the FE and NE groups. Despite comparable network size, there were notable differences between bacterial and fungal interactions between the two groups (Fig. 4d, Fig. S6c-d). For example, in the FE group, four fOTUs, fOTU141 Candida palmioleophila, fOTU2 Candida palmioleophila, fOTU9 Aspergillus spp. and fOTU3 Sordariomycetes spp. showed strong mutual positive correlations in a subnetwork (module 1), which together exhibited co-exclusive relationships with most other fOTUs (Fig. 4c, Fig. S6b). This module was however absent in the network for NE group. On the other hand, another subnetwork, consisting of the mutual co-occurrence relationships between bOTU29 Rothia mucilaginosa, bOTU226 Veillonella spp., bOTU2 Prevotella melaninogenica, bOTU15 Prevotella spp. and bOTU5 Streptococcus spp. (module 2), was specifically present in the network for NE group. Again, network 
272

273

reconstruction using a balanced sample size $(n=26)$ indicated that the different networks observed were not related to sample size (Fig. S7b). Network reconstruction using Spearman correlation mostly recapitulated the findings using SparCC (FDR $\mathrm{p}<0.2$, Fig. S8), indicating the differences in interaction network were robust to the algorithm used.

Correlations of bacterial and fungal microbiome with inflammatory mediators in

\section{$\underline{\text { COPD }}$}

To investigate interactions between bacterial and fungal microbiome and host response, we performed correlation analysis between bacterial, fungal OTUs and inflammatory mediators on a subset of 40 COPD patients with all data available. Fifty-nine correlations were found between blood or sputum mediators and bacterial or fungal OTUs (FDR p<0.20, Fig. 5). Of them, blood IL-6 was negatively correlated with bOTU8 Lactobacillales spp. and bOTU12 Porphyromonas spp. that were the hub OTUs in healthy controls, while it was positively correlated with fOTU8 Cryptococcus spp. that was present in the COPD network. Sputum IL-6 was negatively correlated with bOTU5 Streptoccocus spp. and bOTU29 Rothia mucilaginosa that was part of the module 1, and positively correlated with fOTU5 Aspergillus spp. Sputum IL-8 was positively correlated with fOTU3 Sordariomycetes spp., fOTU9 Aspergillus spp., fOTU15 Leucosporidium scottii and fOTU85 Aspergillus penicillioides, the former two being part of module 2 featured in the network for FE group. We were not able to perform the sub-analysis for frequent and 
294

295

non-frequent exacerbators separately due to the small number of patients in each subgroup with available mediator measurements.

\section{Discussion}

Here we reported the first simultaneous characterization of the airway bacterial and fungal microbiome in COPD. The airway mycobiome was predominated by the phylum Ascomycota over Basidiomycota, consistent with observations in the airways of bronchiectasis and cystic fibrosis $(17,18,38)$ but opposite to one study in asthma(15). We observed significant increases of pathogenic fungal taxa including Candida, Cryptococcus and Schizophyllum in COPD patients. Members of Schizophyllum and Aspergillus participate in invasive infections and provoke host immune recognition(39). Cryptococcus is known to interact with airway epithelium and lead to enhanced allergic inflammation(40). Overall there were greater community shifts for fungi than bacteria in COPD patients versus controls, and in FE versus NE. Supervised learning analysis identified a set of bacterial and fungal OTUs that together showed the optimal discriminatory potential for COPD patients and the frequent exacerbator phenotype, although cross-validation of these features in independent cohorts is warranted.

Importantly, there was a significant negative correlation between bacterial and fungal alpha diversity, both of which altered in opposite directions between COPD and healthy subjects and between FE and NE. Accordingly, individual bacterial and fungal 
OTUs showed disproportionately higher co-exclusive than co-occurrence relationships with each other. In particular, commensal bacterial taxa such as Prevotella and Veillonella exhibited inverse relationships with pathogenic fungal taxa such as Candida palmioleophila and Aspergillus spp. These results support the notion that there was a delicate balance between bacterial and fungal communities in the airways. The disruption of such community balance, characterized by the loss of commensal bacterial taxa and enriched pathogenic fungal taxa, is implicated in COPD pathogenesis $(41,42)$.

We observed distinct patterns of bacterial-fungal interactions both between COPD patients and healthy controls and between FE and NE. In particular, there was an enhanced and more sophisticated microbial interaction in COPD compared to healthy controls, which reflected a more active crosstalk between members of microbiome in response to altered local airway environments in disease. In healthy state, the airway microbiome was dominated by commensal bOTUs that mostly exhibited co-occurrence interactions. In COPD, additional B-F and F-F interactions were involved. While a higher number of F-F interactions were positive, a larger proportion of B-F interactions were negative, a finding that coincides with one recent study on 334 the gut fungal microbiome in colorectal cancer(43). Thus the co-occurrence intrakingdom and co-exclusive interkingdom interactions may be a signature for disease-associated human microbiome in general. This is also in align with the 
disruption of normal bacterial communities may provide pathogenic fungi with a favorable condition for intra-fungi interaction in COPD. Furthermore, several pro-inflammatory mediators such as blood and sputum IL-6 and sputum IL-8 that are known to associate with lung microbiome(4), were negatively correlated with commensal bOTUs in the health-related network, and positively correlated with disease-associated pathogenic fOTUs. Thus the perturbation of ecological interactions in COPD was also associated with increased airway and systemic inflammations.

There were also important differences in bacterial-fungal interactions between FE and NE. The most remarkable difference was the disappearance of five mutually co-occurring commensal bOTUs (module 2) and emergence of four mutually co-occurring pathogenic fOTUs (module 1) in FE. Our results suggest that there was associated with enhanced airway inflammation. The emergence of pathogenic fungi in particular Candida palmioleophila and Aspergillus spp. could be a marker for the and together lead to the accelerated disease progression and increased vulnerability to subsequent exacerbations. 
with different demographic background is warranted to validate our findings. Second, targeted amplicon sequencing has insufficient resolution in species-level identification, in particular for the fungal population with a lack of well-characterized reference database(6). Despite the attempt to improve the fungal taxonomy assignment using phylotyping algorithm, the fungal taxa that can be assigned to genus or species level remain limited. Third, due to limited sputum available, inflammatory mediators were characterized only for a subset of patients and not for healthy subjects, which limits our ability to perform more detailed analysis on host-microbiome interactions between COPD and healthy subjects and within different patient subgroups.

\section{Interpretation}

In summary, we characterized the collective airway bacterial and fungal microbiome in COPD. We showed that the disruption of airway community balance, characterized by the enriched pathogenic fungal taxa over commensal bacterial taxa, is implicated in COPD and associated with airway inflammation. The airway mycobiome is an important cofactor mediating pathogenic infection and airway inflammation, and should be taken into account when assessing the role of airway microbiome in COPD.

\section{Acknowledgements}

Author contributions: $\mathrm{HZ}$ and $\mathrm{ZW}$ had full access to all of the data in the study and takes responsibility for the integrity of the data and the accuracy of the data analysis.

HL, RC and ZW conceived and designed the study. NC, XT, ZHL, ZYL and JS 
382 analysis and interpretation of data and in writing the manuscript. version of the manuscript.

Financial disclosures: The authors declare that they have no competing interests. processed the sputum samples, performed DNA extraction and library preparation. HL, YH and ZW performed all data analysis. RC and HZ supervised the study. ZW wrote the manuscript. All authors provided critical comments and approved the final

Funding: This work was supported by the National Key R\&D Program of China (2017YFC1310600), the National Natural Science Foundation of China (NSFC31970112), and the National Projects of Major Infectious Disease Control and Prevention (2017ZX10103011). Funders had no role in study design, collection, 


\section{References}

1. Shaw JG, Vaughan A, Dent AG, O'Hare PE, Goh F, Bowman RV, Fong KM, Yang IA. 2014. Biomarkers of progression of chronic obstructive pulmonary disease (COPD). Journal of thoracic disease 6:1532-1547.

2. Patel IS, Seemungal TA, Wilks M, Lloyd-Owen SJ, Donaldson GC, Wedzicha JA. 2002. Relationship between bacterial colonisation and the frequency, character, and severity of COPD exacerbations. Thorax 57:759-764.

3. Wilkinson TM, Patel IS, Wilks M, Donaldson GC, Wedzicha JA. 2003. Airway bacterial load and FEV1 decline in patients with chronic obstructive pulmonary disease. American journal of respiratory and critical care medicine 167:1090-1095.

4. Wang Z, Bafadhel M, Haldar K, Spivak A, Mayhew D, Miller BE, Tal-Singer R, Johnston SL, Ramsheh MY, Barer MR, Brightling CE, Brown JR. 2016. Lung microbiome dynamics in COPD exacerbations. Eur Respir J 47:1082-1092.

5. Einarsson GG, Comer DM, Mcllreavey L, Parkhill J, Ennis M, Tunney MM, Elborn JS. 2016. Community dynamics and the lower airway microbiota in stable chronic obstructive pulmonary disease, smokers and healthy non-smokers. Thorax 71:795-803.

6. Underhill DM, Iliev ID. 2014. The mycobiota: interactions between commensal fungi and the host immune system. Nature reviews. Immunology 14:405-416.

7. Nguyen LD, Viscogliosi E, Delhaes L. 2015. The lung mycobiome: an emerging field of the human respiratory microbiome. Front Microbiol 6:89.

8. Huang YJ, Sethi S, Murphy T, Nariya S, Boushey HA, Lynch SV. 2014. Airway microbiome dynamics in exacerbations of chronic obstructive pulmonary disease. J Clin Microbiol 52:2813-2823.

9. Pragman AA, Lyu T, Baller JA, Gould TJ, Kelly RF, Reilly CS, Isaacson RE, Wendt CH. 2018. The lung tissue microbiota of mild and moderate chronic obstructive pulmonary disease. Microbiome 6:7.

10. Sze MA, Dimitriu PA, Hayashi S, Elliott WM, McDonough JE, Gosselink JV, Cooper J, Sin DD, Mohn WW, Hogg JC. 2012. The lung tissue microbiome in chronic obstructive pulmonary disease. Am J Respir Crit Care Med 185:1073-1080.

11.Sze MA, Dimitriu PA, Suzuki M, McDonough JE, Campbell JD, Brothers JF, Erb-Downward JR, Huffnagle GB, Hayashi S, Elliott WM, Cooper J, Sin DD, Lenburg ME, Spira A, Mohn WW, Hogg JC. 2015. Host Response to the Lung Microbiome in Chronic Obstructive Pulmonary Disease. Am J Respir Crit Care Med 192:438-445.

12. Leitao Filho FS, Alotaibi NM, Ngan D, Tam S, Yang J, Hollander Z, Chen V, FitzGerald JM, Nislow C, Leung JM, Man SFP, Sin DD. 2018. Sputum Microbiome is Associated with 1-Year Mortality Following COPD Hospitalizations. Am J Respir Crit Care Med.

13. Sinha R, Weissenburger-Moser LA, Clarke JL, Smith LM, Heires AJ, Romberger DJ, LeVan TD. 2018. Short term dynamics of the sputum microbiome among COPD patients. PLoS One 13:e0191499.

14. Wang Z, Singh R, Miller BE, Tal-Singer R, Van Horn S, Tomsho L, Mackay A, Allinson JP, Webb AJ, Brookes AJ, George LM, Barker B, Kolsum U, Donnelly LE, Belchamber K, Barnes PJ, Singh D, Brightling CE, Donaldson GC, Wedzicha JA, Brown JR, Copdmap. 2018. Sputum microbiome temporal variability and dysbiosis in chronic obstructive pulmonary disease exacerbations: an analysis of the COPDMAP study. Thorax 73:331-338.

15.van Woerden HC, Gregory C, Brown R, Marchesi JR, Hoogendoorn B, Matthews IP. 2013. Differences in fungi present in induced sputum samples from asthma patients and non-atopic 
controls: a community based case control study. BMC Infect Dis 13:69.

16. Mac Aogain M, Chandrasekaran R, Lim AYH, Low TB, Tan GL, Hassan T, Ong TH, Hui Qi Ng A, Bertrand D, Koh JY, Pang SL, Lee ZY, Gwee XW, Martinus C, Sio YY, Matta SA, Chew FT, Keir HR, Connolly JE, Abisheganaden JA, Koh MS, Nagarajan N, Chalmers JD, Chotirmall SH. 2018. Immunological corollary of the pulmonary mycobiome in bronchiectasis: the CAMEB study. Eur Respir J 52.

17. Kramer R, Sauer-Heilborn A, Welte T, Guzman CA, Abraham WR, Hofle MG. 2015. Cohort Study of Airway Mycobiome in Adult Cystic Fibrosis Patients: Differences in Community Structure between Fungi and Bacteria Reveal Predominance of Transient Fungal Elements. J Clin Microbiol 53:2900-2907.

18. Delhaes L, Monchy S, Frealle E, Hubans C, Salleron J, Leroy S, Prevotat A, Wallet F, Wallaert B, Dei-Cas E, Sime-Ngando T, Chabe M, Viscogliosi E. 2012. The airway microbiota in cystic fibrosis: a complex fungal and bacterial community--implications for therapeutic management. PLoS One 7:e36313.

19. Cui L, Lucht L, Tipton L, Rogers MB, Fitch A, Kessinger C, Camp D, Kingsley L, Leo N, Greenblatt RM, Fong S, Stone S, Dermand JC, Kleerup EC, Huang L, Morris A, Ghedin E. 2015. Topographic diversity of the respiratory tract mycobiome and alteration in HIV and lung disease. Am J Respir Crit Care Med 191:932-942.

20. Charlson ES, Diamond JM, Bittinger K, Fitzgerald AS, Yadav A, Haas AR, Bushman FD, Collman RG. 2012. Lung-enriched organisms and aberrant bacterial and fungal respiratory microbiota after lung transplant. Am J Respir Crit Care Med 186:536-545.

21. Dickson RP, Martinez FJ, Huffnagle GB. 2014. The role of the microbiome in exacerbations of chronic lung diseases. Lancet 384:691-702.

22. Wedzicha JA, Brill SE, Allinson JP, Donaldson GC. 2013. Mechanisms and impact of the frequent exacerbator phenotype in chronic obstructive pulmonary disease. BMC Med 11:181.

23. Global initiative for chronic obstructive lung disease (GOLD). Pocket Guide to COPD Diagnosis, Management and Prevention. A Guide for Health Care Professionals. 2019 Report. www.goldcopd.com.

24. Mayhew D, Devos N, Lambert C, Brown JR, Clarke SC, Kim VL, Magid-Slav M, Miller BE, Ostridge KK, Patel R, Sathe G, Simola DF, Staples KJ, Sung R, Tal-Singer R, Tuck AC, Van Horn S, Weynants V, Williams NP, Devaster JM, Wilkinson TMA, Group AS. 2018. Longitudinal profiling of the lung microbiome in the AERIS study demonstrates repeatability of bacterial and eosinophilic COPD exacerbations. Thorax 73:422-430.

25. Caporaso JG, Kuczynski J, Stombaugh J, Bittinger K, Bushman FD, Costello EK, Fierer N, Pena AG, Goodrich JK, Gordon JI, Huttley GA, Kelley ST, Knights D, Koenig JE, Ley RE, Lozupone CA, McDonald D, Muegge BD, Pirrung M, Reeder J, Sevinsky JR, Turnbaugh PJ, Walters WA, Widmann J, Yatsunenko T, Zaneveld J, Knight R. 2010. QIIME allows analysis of high-throughput community sequencing data. Nature methods 7:335-336.

26.Zhou HW, Li DF, Tam NF, Jiang XT, Zhang H, Sheng HF, Qin J, Liu X, Zou F. 2011. BIPES, a cost-effective high-throughput method for assessing microbial diversity. ISME J 5:741-749.

27. Edgar RC, Haas BJ, Clemente JC, Quince C, Knight R. 2011. UCHIME improves sensitivity and speed of chimera detection. Bioinformatics 27:2194-2200.

28. Taylor SL, Leong LEX, Choo JM, Wesselingh S, Yang IA, Upham JW, Reynolds PN, Hodge S, James AL, Jenkins C, Peters MJ, Baraket M, Marks GB, Gibson PG, Simpson JL, Rogers GB. 2018. 
Inflammatory phenotypes in patients with severe asthma are associated with distinct airway microbiology. J Allergy Clin Immunol 141:94-103 e115.

29. Edgar RC. 2010. Search and clustering orders of magnitude faster than BLAST. Bioinformatics 26:2460-2461.

30. Al-Hebshi NN, Nasher AT, Idris AM, Chen T. 2015. Robust species taxonomy assignment algorithm for $16 S$ rRNA NGS reads: application to oral carcinoma samples. J Oral Microbiol 7:28934.

31. Mitra S, Stark M, Huson DH. 2011. Analysis of 16S rRNA environmental sequences using MEGAN. BMC Genomics 12 Suppl 3:S17.

32. Segata N, Izard J, Waldron L, Gevers D, Miropolsky L, Garrett WS, Huttenhower C. 2011. Metagenomic biomarker discovery and explanation. Genome Biol 12:R60.

33. Frank E, Hall M, Witten I. 2016. The WEKA Workbench. Online Appendix for "Data Mining: Practical Machine Learning Tools and Techniques". Morgan Kaufmann, Fourth Edition.

34. Friedman J, Alm EJ. 2012. Inferring correlation networks from genomic survey data. PLoS Comput Biol 8:e1002687.

35. Faust K, Raes J. 2016. CoNet app: inference of biological association networks using Cytoscape. F1000Research 5:1519.

36. Benjamini Y, and Hochberg, Y. 1995. Controlling the false discovery rate - a practical and powerful approach to multiple testing. J R Stat Soc Ser B Methodol 57:289-300.

37. Molyneaux PL, Willis-Owen SAG, Cox MJ, James P, Cowman S, Loebinger M, Blanchard A, Edwards LM, Stock C, Daccord C, Renzoni EA, Wells AU, Moffatt MF, Cookson WOC, Maher TM. 2017. Host-Microbial Interactions in Idiopathic Pulmonary Fibrosis. Am J Respir Crit Care Med 195:1640-1650.

38. Mac Aogain M, Chandrasekaran R, Lim AYH, Low TB, Tan GL, Hassan T, Ong TH, Ng AHQ, Bertrand D, Koh JY, Pang SL, Lee ZY, Gwee XW, Martinus C, Sio YY, Matta SA, Chew FT, Keir HR, Connolly JE, Abisheganaden JA, Koh MS, Nagarajan N, Chalmers JD, Chotirmall SH. 2018. Immunological corollary of the pulmonary mycobiome in bronchiectasis: the CAMEB study. Eur Respir J 52.

39. Ishii KJ, Koyama S, Nakagawa A, Coban C, Akira S. 2008. Host innate immune receptors and beyond: making sense of microbial infections. Cell host \& microbe 3:352-363.

40. Goldman DL, Davis J, Bommarito F, Shao X, Casadevall A. 2006. Enhanced allergic inflammation and airway responsiveness in rats with chronic Cryptococcus neoformans infection: potential role for fungal pulmonary infection in the pathogenesis of asthma. J Infect Dis 193:1178-1186.

41. Iliev ID, Leonardi I. 2017. Fungal dysbiosis: immunity and interactions at mucosal barriers. Nature reviews. Immunology 17:635-646.

42. Marsland BJ, Gollwitzer ES. 2014. Host-microorganism interactions in lung diseases. Nature reviews. Immunology 14:827-835.

43. Coker OO, Nakatsu G, Dai RZ, Wu WKK, Wong SH, Ng SC, Chan FKL, Sung JJY, Yu J. 2018. Enteric fungal microbiota dysbiosis and ecological alterations in colorectal cancer. Gut. 


\section{Figure Legends}

Figure 1. Heatmaps showing the major bacteria and fungi genera ( $>1 \%$ average abundance) in COPD patients and healthy controls. Each column represents an individual grouped first by healthy or COPD subjects and then clustered by bacterial or fungal microbiota composition. The rows on the top represent demographic factors.

\section{Figure 2. Airway bacterial and fungal composition between COPD patients and}

healthy controls. Shannon index for (a) bacterial and (b) fungal microbiome in COPD patients and healthy controls. Beta diversity was assessed based on unweighted UniFrac distances and plotted in PCoA. (c) The AUC curves for the random forest models in separating COPD and healthy groups using the LEfSe-selected bacterial, fungal and their combined OTUs.

Figure 3. Airway bacterial and fungal composition between COPD frequent and non-frequent exacerbators. Shannon index for (a) bacterial and (b) fungal microbiome in COPD frequent (FE) and non-frequent exacerbators (NE). Beta diversity was assessed based on unweighted UniFrac distances and plotted in PCoA. (c) The AUC curves for the random forest models in separating FE and NE using the LEfSe-selected bacterial, fungal and their combined OTUs.

Figure 4. Airway bacterial and fungal interaction networks. (a) The number of significant bacterial-bacterial (B-B), bacterial-fungal (B-F) and fungal-fungal (F-F) 
545

interactions in the networks of healthy controls, COPD patients, the subgroup of COPD patients with healthy-matched sample size (COPD-match), non-frequent exacerbators (NE), frequent exacerbators (FE) and the subgroup of $\mathrm{NE}$ with FE-matched sample size $(\mathrm{p}<0.05)$. (b) Venn diagram for the shared and unique B-B, B-F and F-F interactions between COPD patients and healthy controls, and between FE and NE. (c-d) Bacterial and fungal interaction networks for healthy controls and COPD patients (c), and for NE and FE (d). Nodes were shaped by bacterial or fungal OTUs, and colored by their fold changes in COPD versus healthy groups or in FE versus NE groups. The size of the node is proportional to its degree of connectivity. Edges were colored by co-occurrence (blue) and co-exclusive (red) interactions. Edge width is proportional to the absolute correlation score. Only significant interactions with SparCC correlation $>0.3$ were shown for visualization purpose. Module 1 is highlighted in red dotted ellipse. Module 2 is highlighted in blue ellipse. The full interaction networks are in Fig. S6.

\section{Figure 5. Interaction network between airway bacterial and fungal microbiome}

and host inflammatory mediators. Nodes were shaped by bacterial or fungal OTUs or inflammatory mediators. Bacterial and fungal OTUs were colored by their fold changes in COPD patients versus healthy controls. The size of the node is proportional to its degree of connectivity. Edges were colored by co-occurrence (blue) and co-exclusive (red) interactions. Edge width is proportional to the absolute correlation score in Spearman correlation (FDR p<0.20). Only correlations between 
567 bacterial/fungal OTUs and host inflammatory mediators were shown for visualization

568 purpose.

Historical exacerbation

\begin{tabular}{llll} 
Healthy & COPD & frequency & \\
\cline { 3 - 4 }$(n=29)$ & $(n=84)$ & NE group & FE group
\end{tabular}

$(\mathrm{n}=52) \quad(\mathrm{n}=27)$

\begin{tabular}{lllll}
\hline & 44.28 & 64.55 & & \\
Age, mean (SD) & & & & \\
& $(23.10)$ & $(8.83) * * *$ & & \\
Gender, & $21 / 8$ & & & \\
n(male/female) & & $81 / 3 * * *$ & $50 / 2$ & $25.76)$ \\
Smoking, n(yes/no) & $9 / 20$ & $74 / 7 * * *$ & $45 / 7$ & $23 / 0$ \\
FEV1\% predicted, & & & & \\
mean (SD) & NA & $51.11(23.46)$ & $55.63(23.54)$ & $41.71(19.91)^{*}$ \\
ICS+LABA, n(yes/no) & NA & $51 / 33$ & & \\
\hline
\end{tabular}

570 FEV1: forced expiratory volume in 1s. ICS+LABA: Combination of inhaled

571 corticosteroid and long-acting bronchodilators. $* * * \mathrm{p}<0.001, * * \mathrm{p}<0.01,{ }^{*} \mathrm{p}<0.05$ 


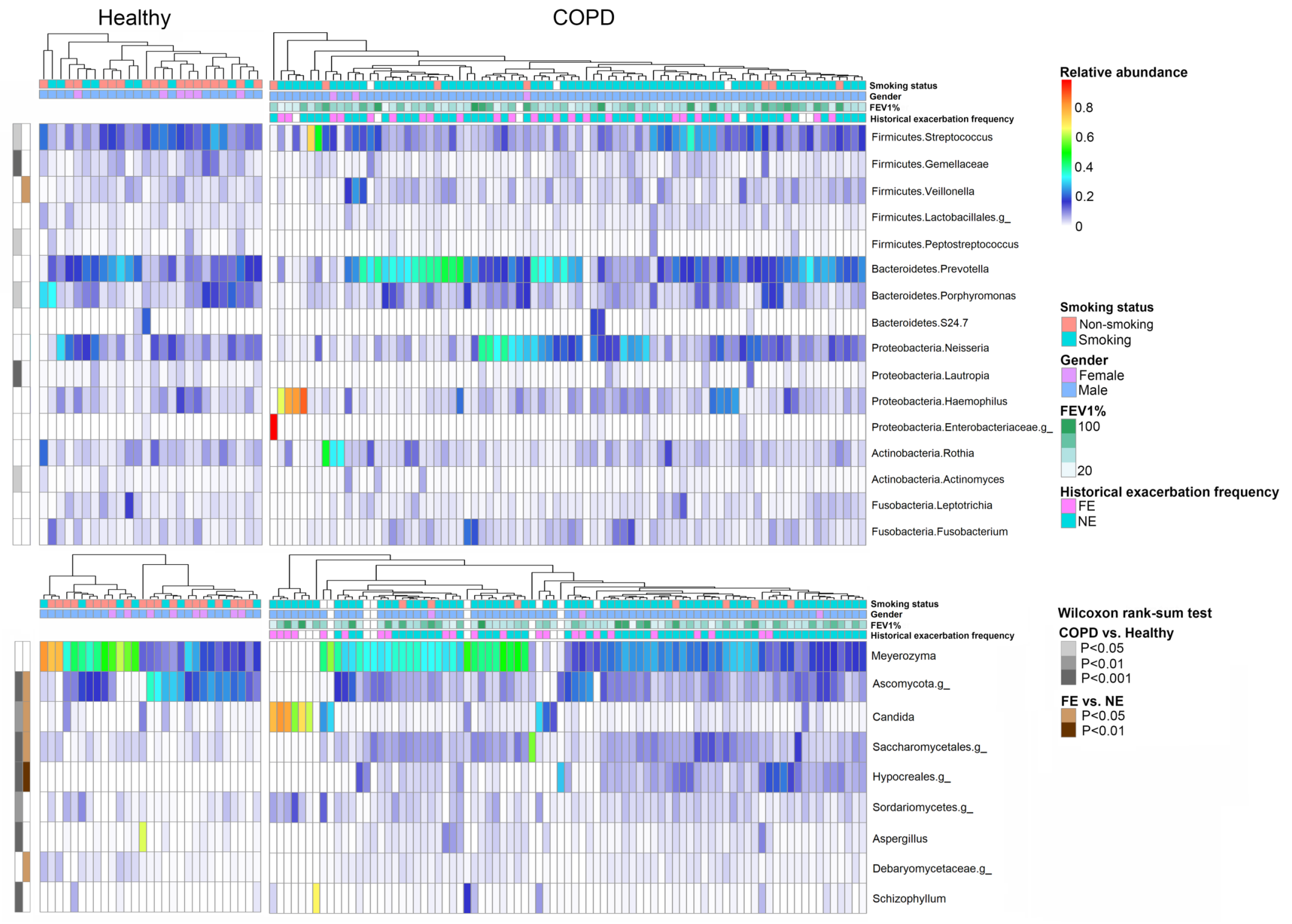



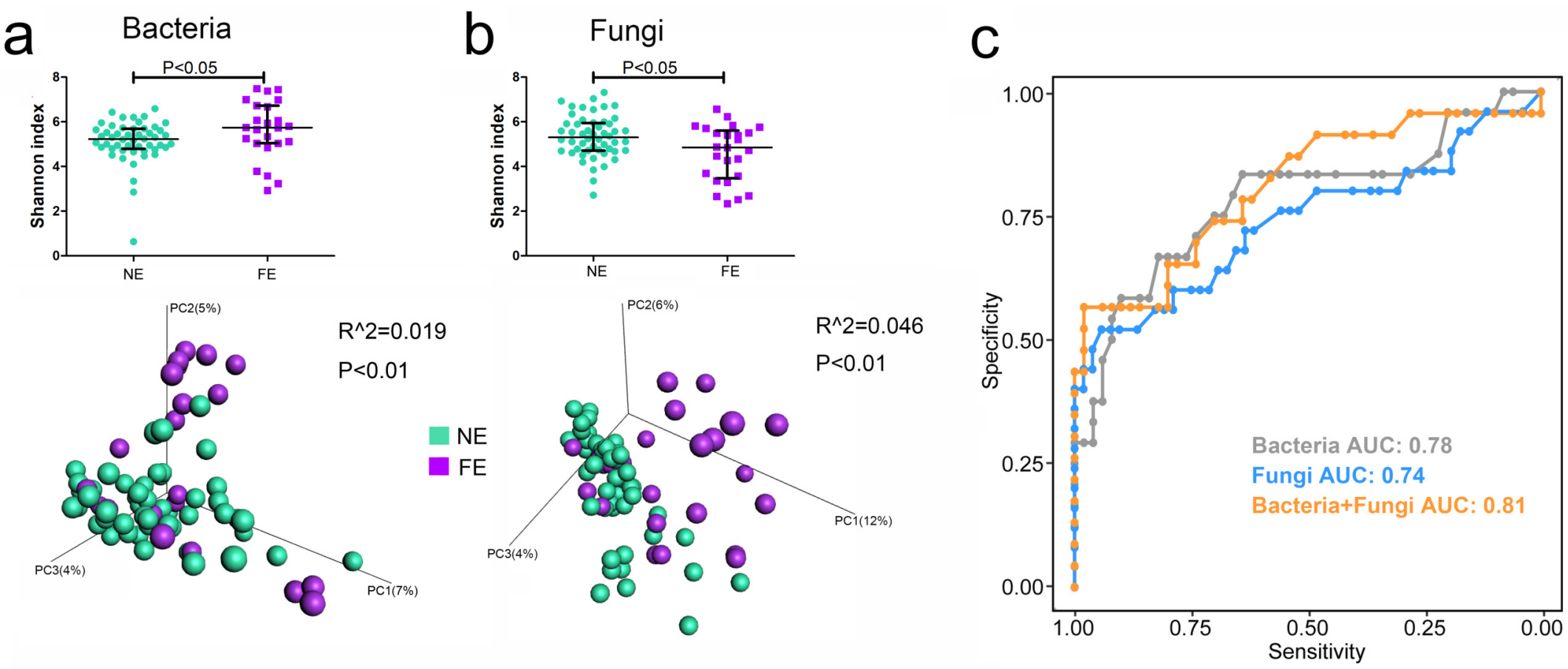
$a$

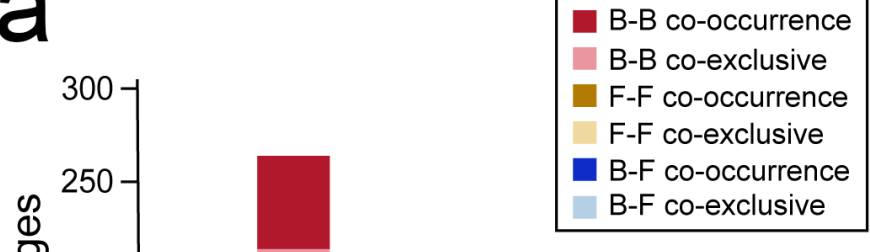

C

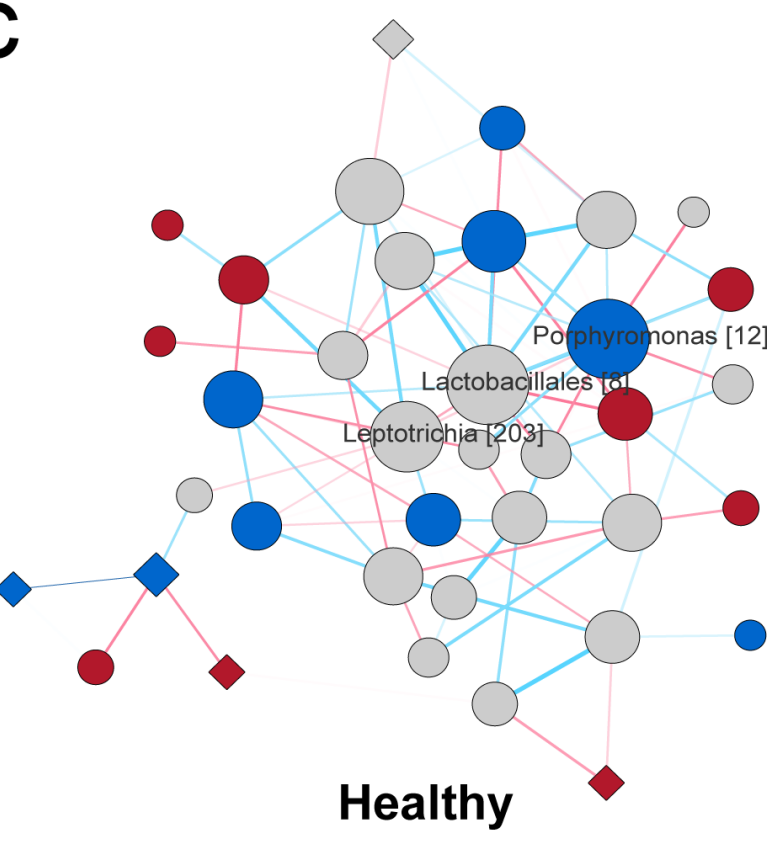

d
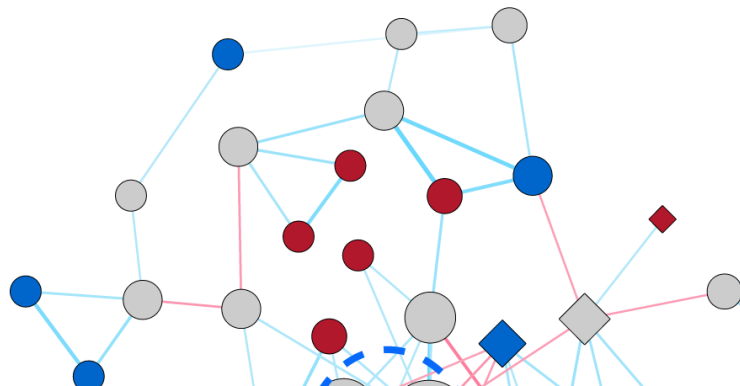

Rothia mucilaginosa 129 ] Prevbtella melaninogenica [2]

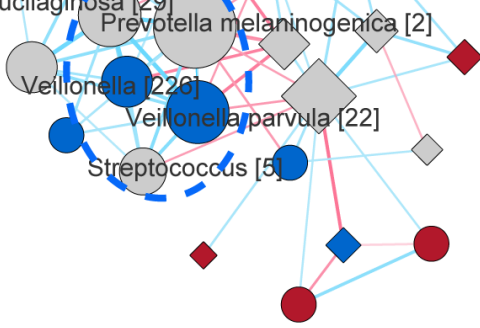

NE
COPD vs Healthy

B-B

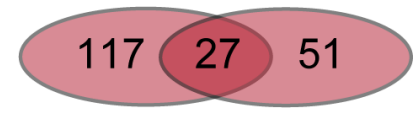

F-F

32

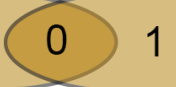

B-F

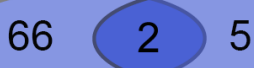

FE vs NE

B-B

$58 \quad 28 \quad 75$

F-F

12712

B-F

42 6 36
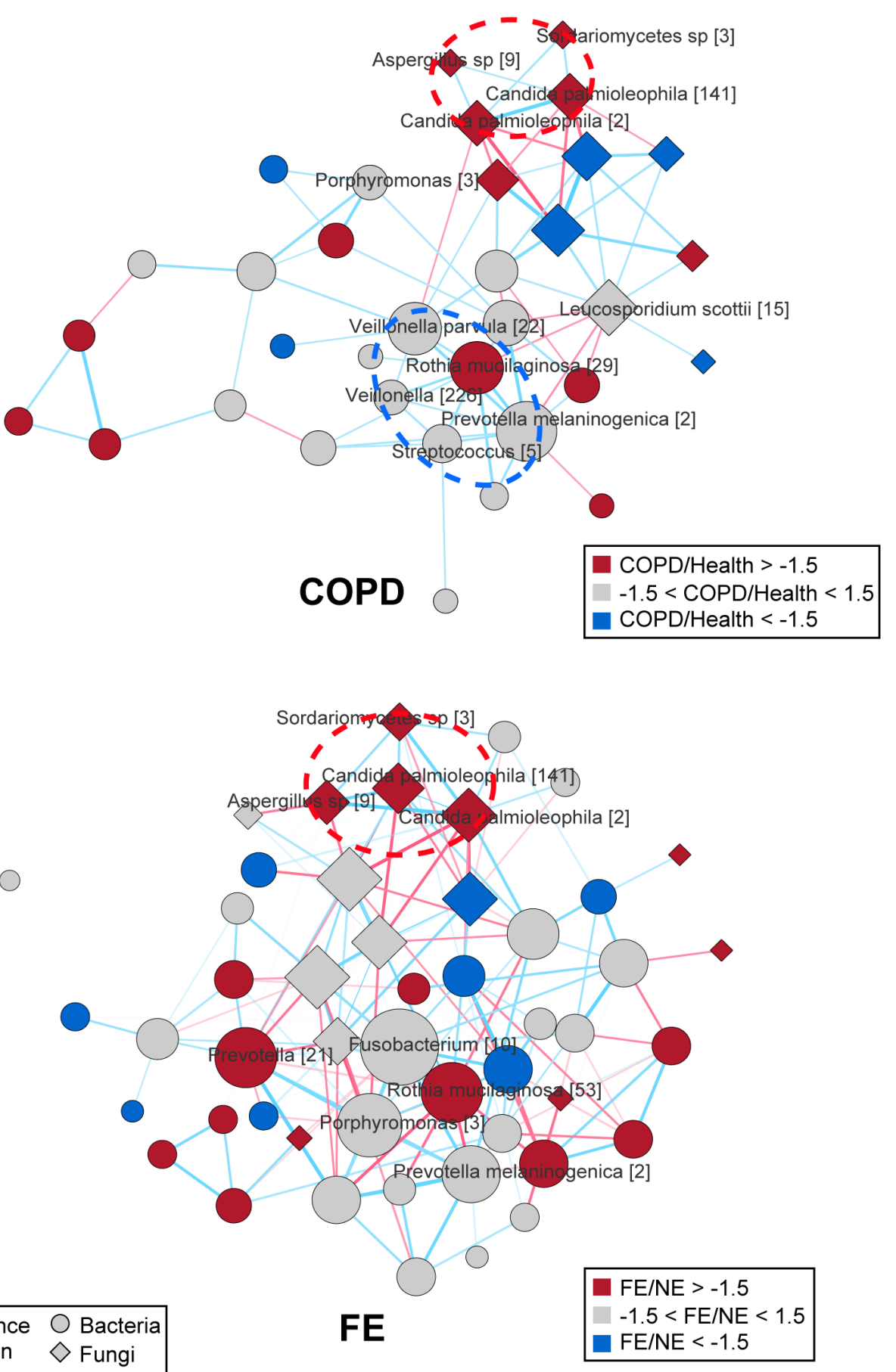
